\title{
El Realejo: una comunidad viva sobre un emplazamiento arque- ohistórico
}

\author{
El Realejo: a living community on a site Historical Archeology \\ Ramiro García Vásquez \\ Biólogo, especialista en Antropología física y \\ Arqueología, consultor, docente \\ Miembro Honorario de la Academia de \\ Geografía e Historia de Nicaragua \\ ID Orcid: http://orcid.org/0000-0003-2186-5452 \\ Sandra Verónica Espinoza Vallejos \\ Especialista en Turismo Cultural, consultora, \\ docente \\ Maestrante en Antropología y Liderazgo Social \\ ID Orcid: http://orcid.org/0000-0003-3239-7615 \\ elidariana07@gmail.com \\ Recibido: 24-11-2018 \\ Aceptado: $10-12-2018$

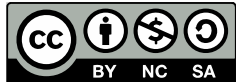 \\ Copyright $\odot 2018$ UNAN-Managua. \\ Todos los Derechos Reservados.
}

mamutsrgv@yahoo.es

\section{Resumen}

La antropología sociocultural de la comunidad de El Realejo, municipio del Departamento de Chinandega es un tópico de gran importancia que necesita ser analizado; se trata de una población emplazada sobre los vestigios arqueohistóricos de lo que fue el primer espacio geográfico colonial de Nicaragua. El conquistador Andrés Niño lo bautizó como Puerto de la Posesión en 1522 y lo anexó formalmente a las propiedades de la Corona española, como parte de las políticas invasoras occidentales, años más tarde lo llamaron Puerto de la Posesión de El Realejo por la fundación de La Villa de El Realejo en 1534 y de ahí deriva su nombre actual. Durante un poco más de trecientos años fue uno de los puertos coloniales más importantes de Centroamerica. En el año 2005 realizamos estudios de carácter antropológico, arqueológico e histórico, en la actual población; obteniendo interesantes datos sobre el emplazamiento colonial, la comunidad viva y la relación entre ambas a través de su Patrimonio Cultural Material e Inmaterial. La riqueza patrimonial del municipio puede ser aprovechada para el desarrollo del Turismo Comunitario Sostenible que permitiría mejorar la economía local a la vez que se protege, conserva, divulga y se logra la puesta en valor de este Patrimonio Cultural del Municipio la Nación.

Palabras claves: Antropología Cultural, Arqueología, Patrimonio Cultural, Turismo Sostenible.

\section{Abstract}

he sociocultural anthropology of the community of El Realejo, municipality of the Department of Chinandega is a topic of great importance that needs to be analyzed; it is a population located on the archeohistorical vestiges of what was the first geographical colonial space of Nicaragua. The conqueror Andrés Niño baptized it as Port of Possession in 1522 and formally annexed it to the properties of the Spanish Crown, as part of the Western invading policies, years later it was called Puerto de la Posesión de El Realejo by the foundation of La Villa de El Realejo in 1534 and hence derives its current name. For a little over three hundred years it was one of the most important colonial ports in Central America. In 2005, we carried out anthropological, archaeological and historical studies in the current population; obtaining interesting data about the colonial location, the living community and the relationship between both through its Material and Intangible Cultural Heritage. The wealth of the municipality can be exploited for the development of Sustainable Community Tourism that would improve the local economy while protecting, preserving, disseminating and achieving the value of this Cultural Heritage of the Nation Municipality.

Keywords: Cultural Anthropology, Archeology, Cultural Heritage, Sustainable Tourism 


\section{Introducción}

El Municipio de El Realejo se localiza entre las coordenadas N: $12^{\circ} 32^{`}$ latitud - W: $87^{\circ} 10^{`}$ longitud sobre la carretera que conduce al Municipio de Corinto y a $11 \mathrm{kms}$ de distancia aproximadamente de la Ciudad de Chinandega, en el departamento de Chinandega. El análisis sobre la Antropología sociocultural e histórica de la comunidad de El Realejo está sustentado en un estudio de carácter científico antropológico en el que se aplicaron técnicas propias del método etnográfico como la observación y la entrevista, técnicas arqueológicas e investigación histórica documental. Según Marvin Harris la antropología cultural se ocupa de la descripción y análisis de las culturas - las tradiciones socialmente aprendidas del pasado y del presente. Tiene una sub disciplina, la etnografía, que se consagra a la descripción sistemática de culturas contemporáneas. La comparación de culturas proporciona la base para hipótesis y teorías sobre las causas de los estilos humanos de vida. (Harris, 2006). Esto precisamente es lo que hacemos en este ensayo; comparar las culturas de la gente de La Villa de El Realejo y la comunidad actual de El Realejo.

Como resultado de la investigación se obtuvo un hiato de información vinculado a la dinámica de vida de las comunidades pretéritas y presentes; los comunitarios fueron muy hospitalarios permitiéndonos observar su vida cotidiana, compartiéndonos los conocimientos que tienen sobre el pasado de su localidad los que les fueron transmitidos de generación en generación y autorizándonos a excavar los sitios seleccionados dentro de este espacio geográfico que fue poblado desde hace más de quinientos años y donde la actual población se emplaza sobre las estructuras arquitectónicas de viviendas e instituciones como iglesias, conventos, la primera aduana, el primer colegio, hospital, cabildo entre otros; de lo que fue el histórico Puerto de la Posesión y la Villa de El Realejo, a quienes los actuales pobladores consideran sus ancestros; fue necesario realizar una investigación histórica documental desde las fuentes primarias como las Crónicas de Indias hasta los historiadores contemporáneos que publican sus obras

sobre este este importante lugar. Para comprobar las fuentes escritas y orales se realizaron excavaciones arqueológicas en dos estructuras arquitectónicas, prime- ro en el Convento San Francisco (1640 - 1810) y posterior en Finca La Batería, propiedad de Don Hilario Alemán (q.e.p.d.); en este sitio se presume que están los vestigios de La Casa de Las Margaritas un prostíbulo que funcionaba como negocio personal de María de Peñalosa y su esposo Rodrigo de Contreras tercer gobernador español en Nicaragua (1535-1550).

En el proceso de trabajo investigativo encontramos evidencias culturales que permitieron corroborar las fuentes históricas que relatan el desarrollo y vida funcional de El Realejo como puerto y como localidad. Lo más relevante de este sitio fue confirmar que la actual población utilizó los cimientos estructurales coloniales para construir sus viviendas; por estas características urbanísticas y arquitectónicas originales es que afirmamos que El Realejo es una comunidad viva sobre un emplazamiento arqueohistórico.

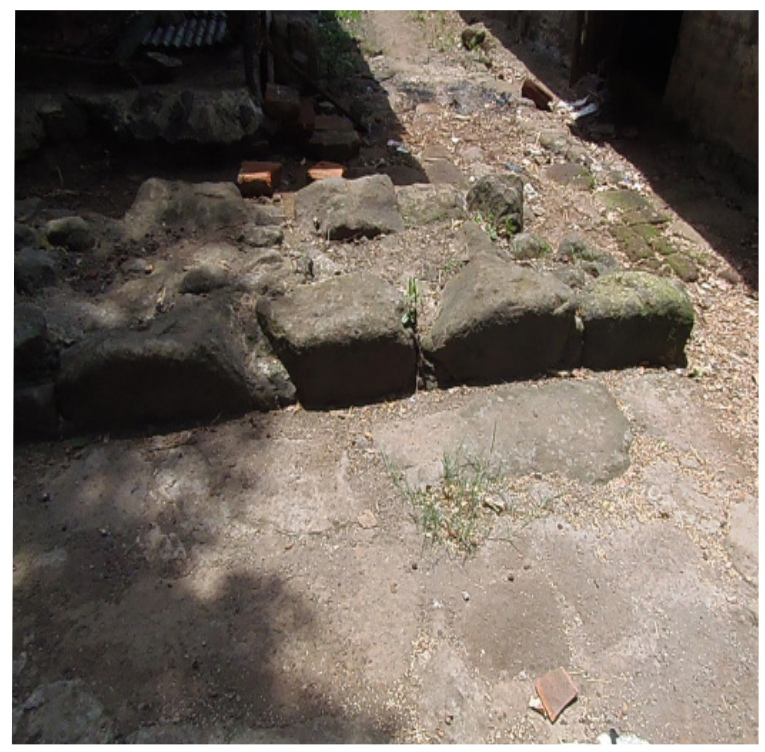

Imagen 1. Obsérvense las viviendas actuales sobre los vestigios históricos. Foto de Sandra Espinoza

\section{El primer espacio geográfico colonial de Nicaragua}

Para reflexionar sobre lo que se considera el primer espacio geográfico del que tomó posesión la colonia en Nicaragua; es importante mencionar que toda la zona occidental del territorio incluida la costera estaba den- 
samente poblada antes de 1522. Las Crónicas de indias mencionan la existencia de tres lenguas principales habladas en lo que hoy es el Departamento de Chinandega: Ulúa en el norte, Nahual alrededor de El Viejo y Oto Mangue-Maribio en el sector oriente, correspondiendo este grupo al espacio geográfico de lo que actualmente es el municipio de El Realejo. (Cronistas 3:3536).

Hurgando la memoria colectiva de la Comunidad Indígena de Sutiaba; entrevistamos al Sr. Roger Montoya, líder de esta colectividad, de 65 años de edad, descendiente de los Sutiaba Maribios y le pedimos que nos contara lo que le transmitieron sus ancestros sobre el Puerto de Icaco y explicó: Nuestros antepasados conocieron y utilizaron este puerto natural para realizar intercambios con los pueblos de Tezoatega, era un punto de encuentro de lanchas y canoas de los Maribios y los Tezoategas. (Montoya, 2016).

La Profesora Linda Newson Cita que al contacto y durante la Colonia Temprana, los nahuas serían unos 67 mil personas en 1522, divididos en los del sur en lo que es hoy Rivas, mejor conocidos como Nicaraos que eran 36 mil aproximadamente, y unos $31 \mathrm{mil}$ al noroeste, alrededor de lo que es hoy es El Viejo, Chinandega y hacia el mar. También dice que la lengua Sutiaba - Tlapaneca del tronco lingüístico Oto - Mangue que en Nicaragua llamaban Maribio ascendía a casi 150 mil personas en 1522. Y que algunas de sus plazas fueron Maçatega, Cindega, Telica, Abangasca, Pangua, Ygualtega, Iguala, Mistega y Astatega. Esto confirma la información que le fue transmitida al líder originario Roger Montoya de generación en generación. Además nos permite afirmar que el Puerto Icaco ya era usado por sus primeros descubridores, que fueron nuestros pueblos originarios. (Newson, 1986).

En 1522 un capitán español llamado Gil González Dávila, llegó a nuestro territorio; siendo este el primer "conquistador" que arribó a Nicaragua después de Cristóbal Colón. Esta expedición era movida por la ambición de encontrar oro, el metal más preciado que ellos buscaban. Dos de los navíos que venían con el Capitán Dávila, estaban dirigidos por Andrés Niño, quien el 27 de febrero de 1522 vio por primera vez un puerto natural en la mar del sur de lo que actualmente es Nicaragua; al que llamó Puerto de la Posesión y que posteriormente sería llamado Puerto de El Realejo. Así lo confirma la siguiente cita:

"Para Nicaragua el hecho más sobresaliente de esta expedición descubridora de Andrés Niño, es el hallazgo y toma de posesión de un punto en la costa noroccidental de nuestro país, que por dicha causa recibió el nombre de Puerto de la Posesión. El hecho tuvo lugar el día 27 de Febrero y sucedió en una islote dentro de la actual bahía de Corinto o de El Realejo; ahí el Capitán Antón Mayor, a nombre de la Corona española desembarcó, cortó ramas y hecho mano a su espada levantando piedras y en pacífica acción y sin contradicción alguna, en presencia de Juan Alanza como escribano dejó consignando en un documento jurídico la formal incorporación de tales tierras a la corona castellana". (Meléndez, 1976). Después de la incorporación formal del puerto a la Corona española, El Realejo se convierte en el primer espacio geográfico colonial de Nicaragua y su historia se basó en una importante función comercial y marítima, vale decir económica durante un poco más de tres siglos (1522 - 1858). En 1534 Pedro de Alvarado Conquistador de Guatemala funda La Villa de El Realejo localizada a orillas del Puerto y a lo largo del Estero Real.

\section{De Puerto de La Posesión a Puerto de El Re- alejo}

Parece ser que el nuevo Puerto colonial, se llamó Puerto de la Posesión desde 1522 hasta 1534, el primer nombre ratifica el hecho de que Andrés Niño tomó formal Posesión de aquel espacio que permitía la navegación, pero el origen de Realejo parece estar en el nombre con que lo llamó Pedro Alvarado; conquistador de Guatemala en el año 1534; cuando navegaba por el Pacífico con rumbo a Perú, pero el mal tiempo le arruinó tres navíos y tuvo que atracar en El Puerto de la Posesión donde había un Astillero para reparar sus barcos. Manuel Rubio Sánchez cita:

A su llegada Pedro de Alvarado llamó al Puerto de la Posesión; Realejo que no es más que un disminutivo de la palabra Real, término usado por los españoles para designar sus campamentos militares. A partir de este año el Puerto de la Posesión fue llamado Puerto de El Realejo. (Sánchez, 1977).

En entrevista realizada a Carlos Pérez poblador de la comunidad y funcionario de la Alcaldía Municipal nos dice: Aquí en la comunidad sabemos que vivimos en un lugar lleno de historia, los muros que hay en los patios de 
las casas son muy antiguos y el nombre del municipio era el que tenía el Puerto histórico, que después dejó de funcionar, porque lo trasladaron a Corinto, por eso nosotros decimos que este pueblo nació desde 1522 y no ha sido abandonado hasta la actualidad vivimos en El Realejo. (Pérez, 2005).

\section{Importancia socioeconómica del Puerto de El Realejo y su abandono}

Parece ser que el nuevo Puerto colonial, se llamó Puerto de la Posesión desde 1522 hasta 1534, el primer nombre ratifica el hecho de que Andrés Niño tomó formal Posesión de aquel espacio que permitía la navegación, pero el origen de Realejo parece estar en el nombre con que lo llamó Pedro Alvarado; conquistador de Guatemala en el año 1534; cuando navegaba por el Pacífico con rumbo a Perú, pero el mal tiempo le arruinó tres navíos y tuvo que atracar en El Puerto de la Posesión donde había un Astillero para reparar sus barcos. Manuel Rubio Sánchez cita: A su llegada Pedro de Alvarado llamó al Puerto de la Posesión; Realejo que no es más que un disminutivo de la palabra Real, término usado por los españoles para designar sus campamentos militares. A partir de este año el Puerto de la Posesión fue llamado Puerto de El Realejo. (Sánchez, 1977).

En entrevista realizada a Carlos Pérez poblador de la comunidad y funcionario de la Alcaldía Municipal nos dice: Aquí en la comunidad sabemos que vivimos en un lugar lleno de historia, los muros que hay en los patios de las casas son muy antiguos y el nombre del municipio era el que tenía el Puerto histórico, que después dejó de funcionar, porque lo trasladaron a Corinto, por eso nosotros decimos que este pueblo nació desde 1522 y no ha sido abandonado hasta la actualidad vivimos en El Realejo. (Pérez, 2005).

\section{Vestigios de una comunidad arqueohistóri- ca. Excavaciones arqueológicas en el Con- vento San Francisco. (2005)}

Hurgando los documentos legales contenidos en las Crónicas y referidos a La Villa de El Realejo, encontramos que en 1764, pasó a ser llamada El Corregimiento de El Realejo, integrado por La Villa de El Realejo, La Villa de Chinandega, La Villa de Chichigalpa y La Villa de El Viejo. La descripción que se hace de El Realejo dice: El Corregimiento de El Realejo, se compone de cuatro poblaciones: La Villa que está junto al Pueblo, que fue poblado de españoles con Alcalde, Cabildo y Regimiento, Dos Conventos el de San Francisco y La Merced y un Hospital de San Juan de Dios. Y al presente sólo se hallan las ruinas y vestigios de los templos y casas y sólo la habitan unos pocos negros, y mulatos carpinteros. El pueblo de Chinandega, el de El Viejo y Chichigalpa son de indios mesclados con ladinos de todas esferas, producidos de las familias que se despoblaron de El Realejo y todas estas poblaciones están en distancias de cuatro leguas y en su entorno hay muchas estancias de ganado vacuno, y algunas haciendas de trapiches de hacer de azúcar. (Sánchez, 1977).

En esta cita encontramos los orígenes de la etnología y la antropología económica de los actuales municipios de El Realejo, Chinandega, Chichigalpa y El Viejo. Además tenemos una reconstrucción de la funcionalidad que tuvieron las estructuras arquitectónicas históricas que se observan en casi todos los solares de las viviendas de los actuales pobladores de la comunidad de El Realejo, que funcionaron como instituciones religiosas como los conventos Franciscanos y Mercedarios y públicas como El Cabildo, La Aduana, La Escuela y El Hospital. Con el objetivo de corroborar que en la comunidad de El Realejo se conservan los vestigios de uno de los primeros conventos coloniales de Nicaragua, se realizó excavaciones arqueológicas en dos espacios internos de la estructura religiosa denominada Convento San Francisco de El Realejo.

El Padre Edgard Zúñiga en su obra Historia Eclesiástica de Nicaragua se refiere a la ausencia de conventos en La Villa de El Realejo para el año de 1621, cita: En la villa y Puerto de El Realejo, Provincia de Nicaragua a trece días del mes de Febrero de 1621, ante el capitán Cristóbal de Salazar, corregidor de esta villa por SM. Fue leída esta petición; enviar a su majestad (S.M) en su Real consejo de Indias: Que hasta ahora no ha habido ni hay convento alguno fundado con licencia de su majestad y que ha carecido y carece de quien predique la palabra evangélica y acuda a la crianza de buenas costumbres de los niños que aquí nacen. (Zuniga, 1997)”. El padre Zúñiga recoge otra cita referida a la fundación del Convento, dice: "En Enero de 1639 el padre Franciscano Pedro de Zúñiga fue nombrado por el definitorio de la provincia de San Jorge comisario de la 
provincia ante la corte, con la intención de traer más franciscanos a Nicaragua, el Fraile Zúñiga viajó a España y ya de vuelta a nuestras tierras en compañía de 22 frailes en el galeón de San Juan frente a la costa de Cádiz fue asaltado por corsarios franceses el 22 de Julio de 1640 quedando como naufrago en el mar hasta el día 27 cuando llegó a las costas prácticamente desnudo, salvado milagrosamente por la intercepción de nuestra señora del Viejo. Para esta fecha los conventos Franciscanos más importantes de Nicaragua eran: El de la Concepción de Granada, El de Santiago de Jinotepe, El de Santa Ana de Chinandega, El de la Asunción del Viejo, El de la Concepción de Rivas y El San Antonio del Realejo. (Zuniga, 1997).

Para mediados de 1700 el convento pasó a llamarse San Francisco; así lo dice la siguiente cita: "En 1752 el obispo de la diócesis de Nicaragua Marín Bullón y Santa Cruz realizó una gira por todas las iglesias de la provincia, cuando llegó a La Villa de El Realejo que ya para esos días estaba en franco abandono había una parroquia a cargo de un cura y un sacristán Mayor y un Convento o iglesia de San Francisco a cargo de un guardián que atendía por cuaresma. (Zuniga, 1997). Es probable que este Convento estuviera
La población de la comunidad de El Realejo se integró y fue participe del estudio arqueológico, cuatro jóvenes comunitarios de nombre Julio Zelaya Paredes, Víctor Mayorga Juárez, José Salinas y Abdul Pereira Paredes formaron parte del equipo de asistencia en las excavaciones y nos compartieron sus memorias míticas sobre el edificio; afirmando que era un convento y que se conectaba con la iglesia San Benito por un túnel subterráneo, donde habían tesoros que buscaban los piratas y que por eso lo habían sellado. Doña Benita (q.e.p.d) vento, mi casa está sobre ellos y también la de los vecinos, si camina en los solares puede verlos y restos de tejas, botellas de vino todo del tiempo cuando El Realejo era un Puerto. (Benita, 2005).

Se realizaron dos excavaciones, una en el Altar Mayor donde se encontraron vestigios arquitectónicos correspondientes al piso del edificio de la primera etapa constructiva del edificio, en este piso se encontró evidencia de combustión confirmando el dato histórico que dice que el primer edificio se quemó y tuvo que ser reconstruido con mejores materiales; también encontramos un rasgo funerario consistente en restos óseos humanos asociado al sexo femenino y auxiliándonos por la Antropología física se logró a determinar que era una joven entre 20 a 25 años de edad, con una estatura de 1.50 a $1.55 \mathrm{~m}$. aproximadamente. Por las características biológicas de sus dientes en forma de pala inferimos que era perteneciente a la población mestiza y fue sepultada en un espacio de privilegio dentro del edificio, sugiriendo que pudo haber estado relacionada con el servicio religioso en el templo.

El siguiente espacio afectado por la excavación fue el presbiterio del convento donde se encontraron tres rasgos funerarios que de 1800 , pues en 1823 ya estaba en ruinas y no tenía funcionamiento religioso, esto se comprueba con la cita:

tenía su vivienda colindante con los vestigios arqueohistóricos, le hicimos una entrevista en el año 2005 cuando tenía 65 años de edad, ella nos compartió lo siguiente: A mí me contaron que era un convento muy grande, lo que usted ve cercado con la maya es solo la parte donde estaba la iglesia pero no era ese el tamaño, aquí donde estamos hay muros del con- contenían fracciones esqueléticas pertenecientes a un infante, una mujer joven y un adulto maduro pasado de la sexta década de vida. Estos individuos fueron sepultados en este espacio después de la segunda etapa constructiva del edificio (1646). La tercera tumba encontrada tiene todas las características de un típico entierro cristiano español, este patrón de enterramiento ya se había registrado en León de Imabite y el Convento San Francisco de Granada. (García, 2010) 
El análisis de Antropología Física "In Situ" infiere que se trata de un hombre adulto pasado de la sexta década de vida, $1.75 \mathrm{mts}$ de altura, sepultado en una caja de madera sellada con clavos, la posición de su cuerpo es viendo al altar mayor, con sus manos debidamente colocadas sobre el tórax y sus pies juntos, con evidencias de afectaciones patológicas en las articulaciones de los huesos largos, enterrado en el tercer espacio del presbiterio del altar mayor como cierto privilegio que se la daba a las personas con estatus social de la época.

Al observar esta tumba Daña Benita que gentilmente todos los días nos preparaba el desayuno, nos comentó que sus abuelos le dijeron que en ese convento estaba enterrado el Alcalde que fue del puerto de El Realejo. Revisando las fuentes bibliográficas encontramos una cita referida a este personaje que dice:

Como vimos, en 1640 el Alférez Pedro Moreno Rubio, fue nombrado vitaliciamente, Depositario General de la Villa y Puerto del Realejo en virtud de haber hecho la mejor oferta para ocupar el puesto. Por el año 1658, según el estipulado, renuncia de dicho empleo en favor de su cuñado José Romero y de su hijo don Tomas Moreno. Acaeciendo en este año la muerte de don Pedro Moreno
Rubio conforme se acostumbraba y se ordenaba las diligencias al respecto se efectuaron. (Sánchez, 1977).

Esta cita nos da la fecha de fallecimiento del Alcalde Vitalicio de El Realejo Don Pedro Moreno Rubio y esta fecha coincide con el período en el cual el templo o Convento San Francisco era el único edificio religioso de El Realejo y por lo tanto el único cementerio o espacio de enterramiento digno para una personalidad como era este español. El hecho que la cita diga que Don Pedro Rubio, delegó el cargo en su hijo nos hace inferir que era un hombre adulto maduro al morir, probablemente de la tercera edad, lo que también coincide con las características biológicas de los restos óseos del espécimen descubierto en la tumba número tres. La cita también menciona que después del descenso de Don Pedro Rubio se actuó conforme se acostumbraba y se ordenaban las diligencias lo que sugiere que le practicaron un entierro típico de su época, religión e importancia social.

Mediante el estudio se corroboró que el espacio religioso fue utilizado como cementerio ya que en esa época todavía no se habían creado los cementerios, además se encontró suficiente evidencias para afirmar que se construyó en dos ocasiones ya que fue destruido por el fuego. Finalmente se verificó que parte de los muros del edificio se encuentran como cimientos de viviendas actuales y que no se ha delimitado completamente el espacio del Convento.

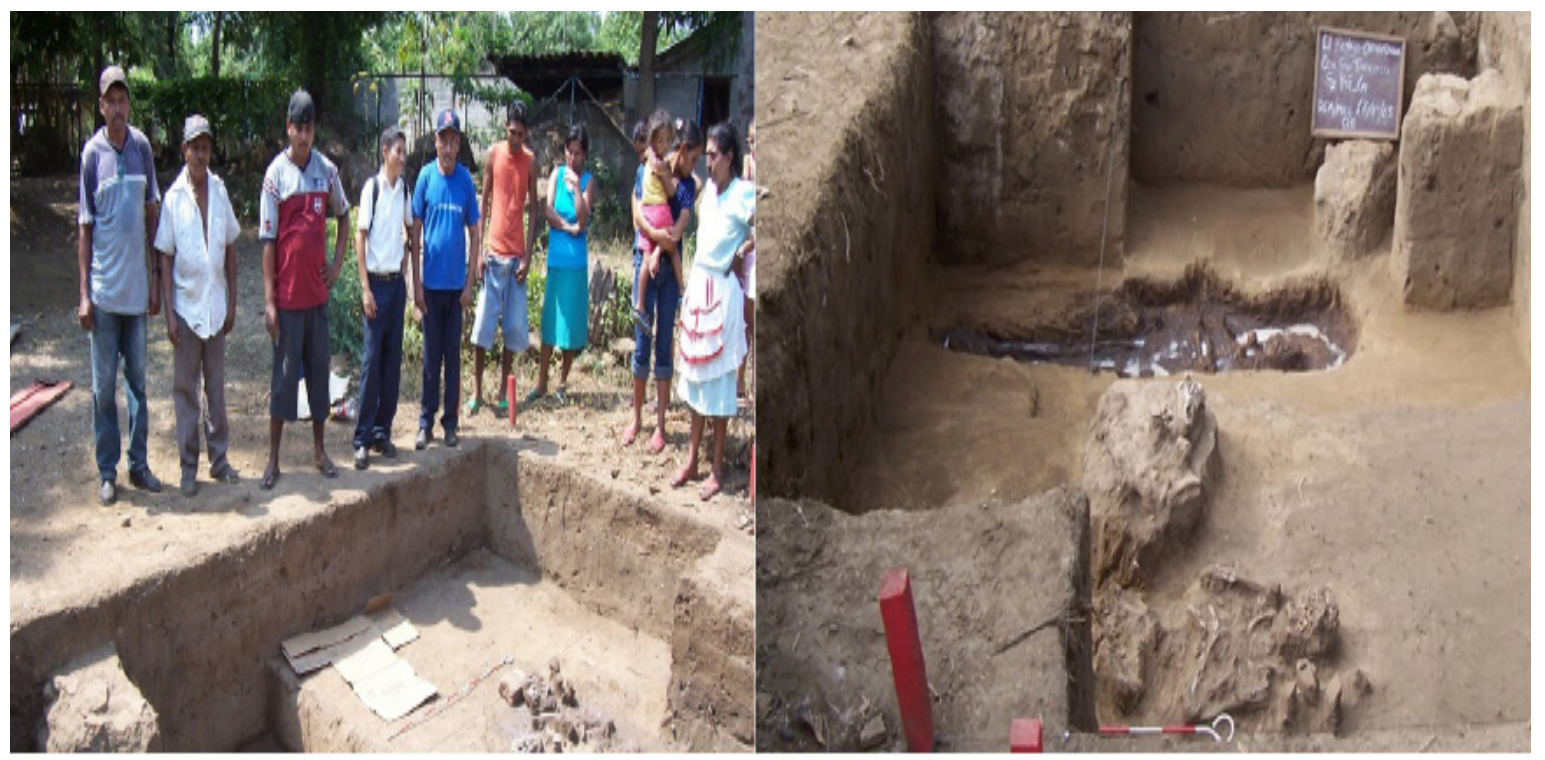

Imagen 2. Cuadriculas de excavaciones con participación de la comunidad, 2005. Foto Ramiro García. 
Descubrimiento de los empedrados del Burdel de Las Pedrarias en El Realejo para continuar hurgando en la Antropología histórica de la comunidad de El Realejo realizamos un estudio arqueológico en un espacio habitacional denominado Finca La Batería, localizada entre las coordenadas Norte: $12^{\circ}, 32$, 12 ". Oeste: $87^{\circ}, 10^{\prime}, 03$ " muy próxima al sitio donde estuvo el muelle del histórico Puerto. La casa tiene fachada colonial con los típicos corredores por los cuatro costados, emplazada en la periferia del poblado, al final de la calle principal de El Realejo. Las excavaciones fueron realizadas en el año 2005 con la autorización del entonces dueño de la propiedad el Sr. Hilario Alemán (q.e.p.d) y toda su amable familia. La casa está construida sobre los cimientos de piedra de una estructura arquitectónica antigua de la época colonial. Para conocer datos del inmueble entrevistamos a Don Hilario y nos contó: Esta Ca- sona fue construida hace 200 años, yo soy de Chichigalpa vine a vivir aquí en 1980, hace unos años me vino a visitar un hombre llamado Ricardo Pasos Marciacq me dijo que era escritor de novela y que esta casa estaba sobre las ruinas de un prostíbulo y que iba a escribir un libro llamado El Burdel de Las Pedrarias, que aquí en los tiempos del puerto traían a las mujeres más lindas de Tezoatega que es El Viejo y que las obligaban a ser prostitutas, eran esclavas y las mataban. Yo no sabía nada de eso pero él me dijo que un gobernador español era el dueño y que el nombre de la vieja casona era La Casa de Las Margaritas. (Alemán, 2005).

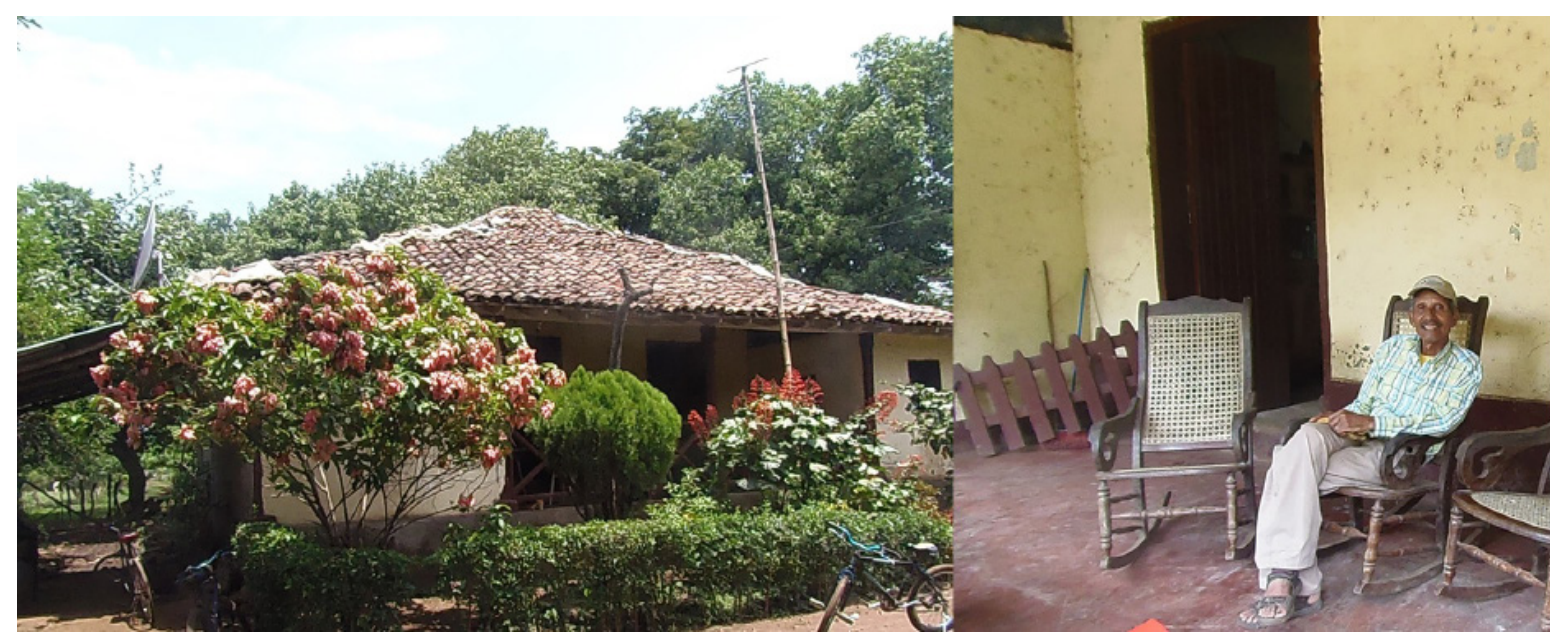

Imagen 3. Don Hilario Alemán (q, e, p, d.) sentado en el alero frontal de la casona de la Finca La Batería. 2017. Foto Sandra Espinoza.

En las fuentes históricas encontramos una cita referida al tercer gobernador de Nicaragua Rodrigo de Contreras y su esposa María de Peñaloza, quienes gobernaron la provincia entre los años de 1535-1550, la cita trata de un juicio enfrentado por el gobernador ante la audiencia e incluso ante la Corona española donde se le acusa de tener un negocio ilegal que consistía en la prostitución, esclavitud y maltrato de mujeres libres del poblado de Tezoatega (El Viejo), para venderlas como prostitutas a los marineros que pasaran por el Puerto de El Realejo. Las citas dicen textualmente: ..." Yten que consiste que un pueblo de Yndios que tiene en administración Rodrigo de Contreras que se dize Teote- ga ques de su suegra se alquilen las indias libres del a marineros es otras personas que están en el puerto del Realejo ques doze leguas de León e una legua del dicho pueblo deste Teotega esto para que se hechen con las tales personas e ansy por vna india hermosa piden a vn precio e por otra que no loes tanto otro precio y si otra persona las alquila le castigan y le hechan pena por ello."

Otro testigo dice: "Ávido venir a esta ciudad al dicho Rodrigo de Contreras de un pueblo ques de la dicha doña María de Peñalosa que está cerca del Realejo." (Bolaños, 1954)

Estas citas históricas afirman que Rodrigo de Contre- 
ras y su mujer María de Peñalosa, pusieron a funcionar un prostíbulo cerca del Puerto de El Realejo, donde obligaban a mujeres jóvenes autóctonas del pueblo de Tezoatega a ser prostitutas, empleando una forma cruel de esclavitud sexual con estas mujeres, y que las tomaban, porque Tezoatega era una encomienda que le pertenecía por herencia a Doña Isabel de Bobadilla mujer de Pedrarias Dávila primer Gobernador de Nicaragua quien se la cedió a su hija Doña María de Peñalosa esposa de Rodrigo de Contreras.

Ricardo Pasos Marciacq, en su novela histórica El Burdel de Las Pedrarias, menciona que existió una casa en el Puerto de la Posesión la que fue construida y arreglada exclusivamente para recibir a los clientes que llegaban a comprar el servicio sexual de las mujeres precolombinas obligadas a ser prostitutas para mantener el negocio del matrimonio Contreras - Peñalosa. En este libro se menciona que la casa estaba a 600 varas del atracadero y que uno de los primeros clientes del negocio fue el adelantado Pedro de Alvarado que llevó más de 100 soldados a comprar el servicio de las mujeres en esta casa de citas.

La investigación arqueológica en la Casa Hacienda La Batería tenía como fin encontrar evidencias que confirmen la información dada por las fuentes históricas y orales a cerca de la existencia del prostíbulo establecido por Rodrigo de Contreras. Se realizaron dos cuadrículas en las que se encontró un piso empedrado característico de las construcciones coloniales principalmente para calles y aleros de las viviendas coloniales, este patrón constructivo ya ha sido registrado arqueológicamente en antigua Panamá y fue usado en algunas calles de la actual ciudad de León, Granada, El Viejo y Chichigalpa en nuestro país. Con el objetivo de agotar la posibilidad de que el empedrado representara un montículo precolombino más específicamente un espacio de enterramiento de nuestros pueblos ancestrales decidimos mover una parte de

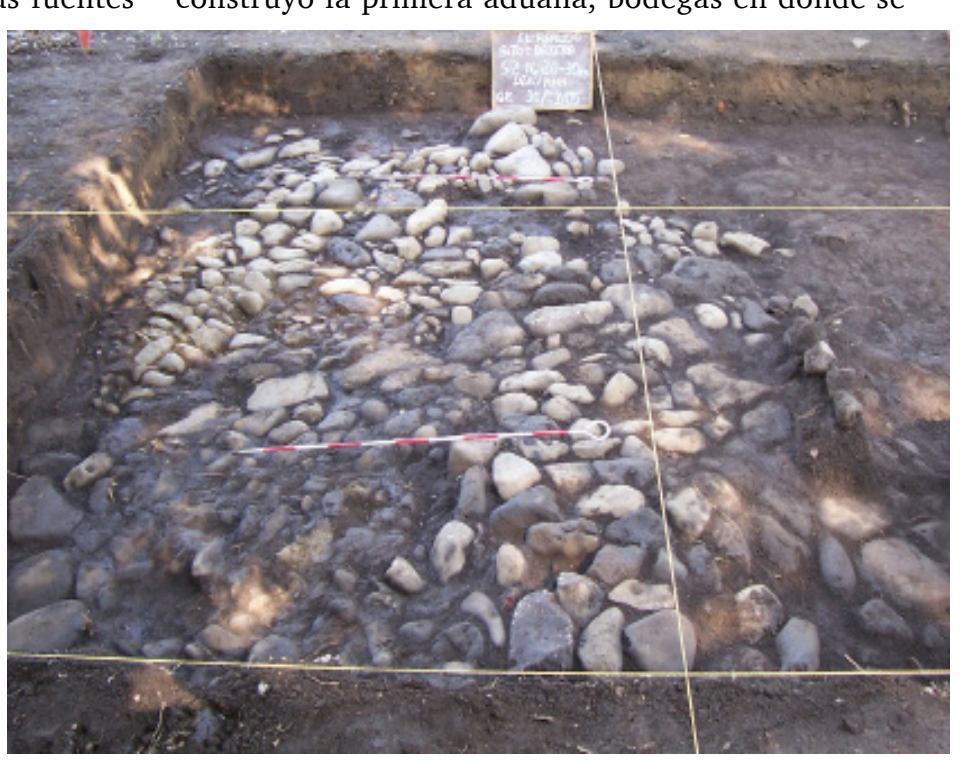

Imagen 4. Empedrado de un alero de la Casa de las margaritas. 2005. Foto Ramiro García.

las piedras y profundizar la excavación lo que sirvió para saber que no existía ningún vestigio cultural prehispánico bajo el empedrado. Durante la excavación solamente se encontraron algunos clavos coloniales probablemente de carretas y fragmentos de porcelana; lo que nos hace inferir que esos materiales son parte de las actividades antrópicas que realizaban en este espacio y que quedaron en el subsuelo antes de la construcción del empedrado.

El estudio nos indica que el espacio investigado es parte del complejo arquitectónico de una casa colonial de grandes dimensiones que se extiende hasta donde actualmente está la casa de La Finca La Batería, es seguro que al realizar investigaciones en todo el sitio podríamos definir los límites y formas constructivas del edificio arquitectónico colonial y posteriormente con áreas definidas podríamos encontrar evidencias de las actividades antrópicas que realizaban en la vivienda.

Por ahora nos limitamos a definir que el sitio en donde está la hacienda La Batería es un yacimiento arqueológico con vestigios culturales y arquitectónicos principalmente coloniales y que sería de mucha importancia para la historia de Nicaragua investigar todo el edificio; al igual que todas las construcciones coloniales de El Realejo; pues representan el estilo urbanístico de la colonia temprana. De acuerdo a las fuentes históricas en este Puerto se construyó la primera aduana, bodegas en donde se 


\section{Puesta en valor del Patrimonio material e inmaterial de El Realejo a través del turismo comunitario}

Según Marvin Harris la Antropología Cultural es el estudio de la humanidad, de los pueblos antiguos y modernos y de sus estilos de vida. La actual comunidad de El Realejo es una población con aproximadamente 1200 viviendas, de todo tipo, (madera, concreto, ranchos de palma), algunas con piso de concreto, ladrillos y tierra, los techos son de zinc, palma y tejas en su gran mayoría. Las construcciones modernas están sobre los cimientos o estructuras arquitectónicas de la época colonial y en muchos lugares aún se pueden observar vestigios de las antiguas construcciones. (BCN, 2017). Estos vestigios arquitectónicos junto a los objetos históricos que se conservan en el subsuelo y toda la memoria colectiva que resguarda la comunidad forman parte del Patrimonio Cultural Material Mueble-Inmueble e Inmaterial de la comunidad de El Realejo. Los pobladores reconocen que sus raíces e identidad están en el antiguo asentamiento histórico, pero necesitan empoderarse de todos estos recursos para que sean los garantes de la protección, conservación y puesta en valor de los bienes patrimoniales. La población de El Realejo necesita crear emprendimientos locales, formar asociaciones comunitarias de la mano de los programas solidarios del Gobierno de Unidad y Reconciliación Nacional, que les permitirán el desarrollo del Turismo Comunitario para transformar el pueblo de El Realejo en un Museo "In Situ", un espacio interpretativo donde la comunidad viva muestre que están asentados sobre los vestigios de sus antepasados. Agustín Santana, 1997 alertaba respecto a la necesidad de un desarrollo integral de lugares con potencial turísticos. El concepto de patrimonio se asocia a la idea de "herencia", ya sea en relación al arte, la arqueología o lo artístico. El desarrollo sustentable aparece como una alternativa a la crisis ambiental - cultural, se evidencia, de hecho, que cualquier escenario cultural puede convertirse en un recurso y producir beneficios, y para ello es necesario establecer mecanismos de interrelación entre el sector público, principalmente gobiernos locales y regionales, el sector empresarial, las instituciones de investigación y formación, y sin lugar a dudas, los propios locales. Se ha de tener en cuenta la cultura en todas las acciones de desarrollo en una triple vertiente: como factor de cohesión social, como factor de diálogo entre los pueblos y como generador de empleo. (Áreas, 2006).

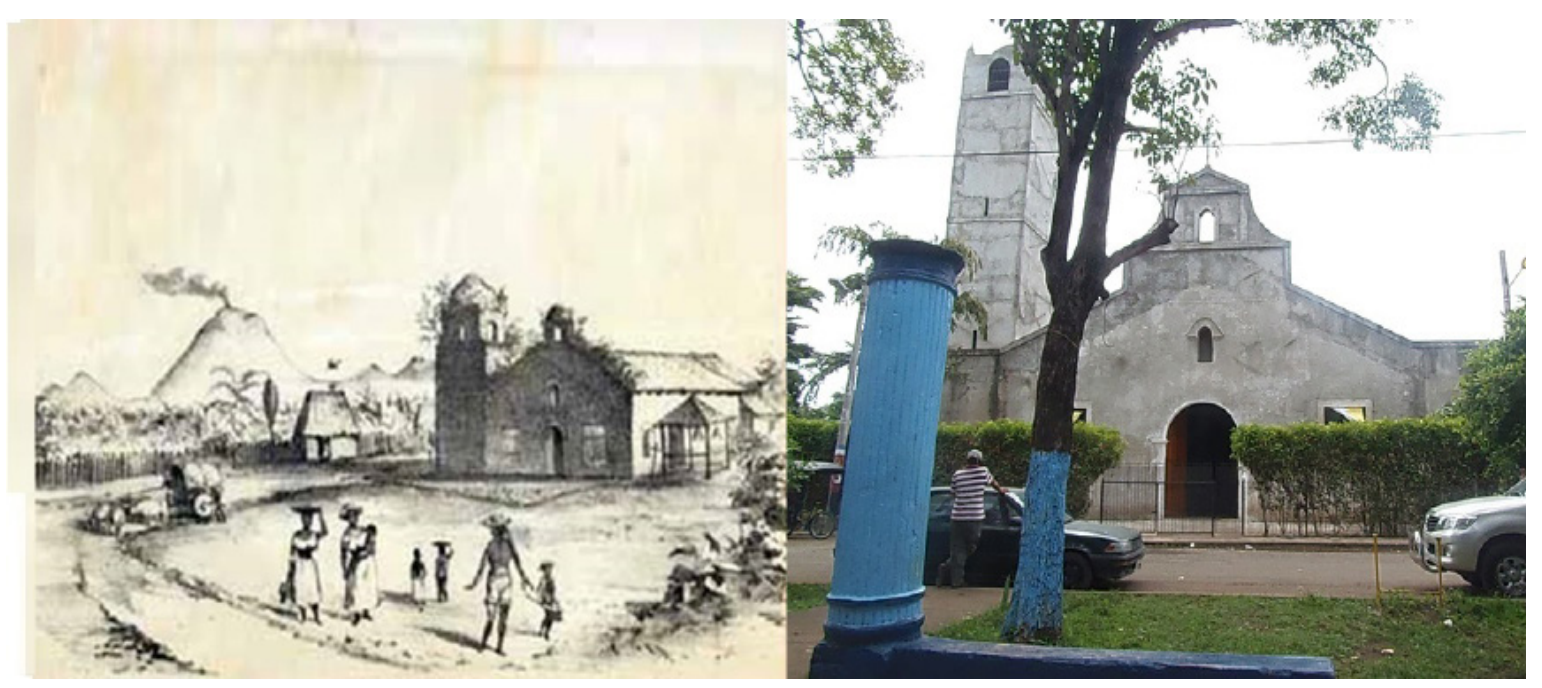

Imagen 5. El Realejo ayer y hoy. Dibujo tomado del Libro Historial de

El Realejo. (Sánchez, 1977). Foto de Sandra Espinoza. 


\section{Conclusión}

Como lo refiere Molano 2008, nadie puede vivir sin recordar y nadie puede vivir tampoco sin los recuerdos de la historia; la historia está allí orientando nuestros juicios a cada instante, formando nuestra identidad, determinando la fuente y toma de conciencia de nuestros valores. Los resultados de los estudios realizados en El Realejo desde la antropología, la etnografía, la arqueología y la historia han permitido corroborar la importancia y el significado antropológico, histórico, cultural y comercial que tiene espacio geográfico de nuestro territorio. Para construir una antropología del

Alemán, H. (25 dE FebreRo de 2005). LA CASA DE LAS MARGARITAS. (R. GARCÍA, ENTREVISTADOR)

ÁREAS, D. L. (2006). SE EVIDENCIA, DE HECHO, QUE CUALQUIER ESCENARIO CULTURAL PUEDE CONVERTIR- SE EN UN RECURSO Y PRODUCIR BENEFICIOS, Y PARA ELLO ES NECESARIO ESTABLECER ME- CANISMOS DE INTERRELACIÓN ENTRE EL SECTOR PÚBLICO, PRINCIPALMENTE GOBIERNOS LOCALES Y REGIONALES, EL. S/C: S/E.

BCN, B. C. (2017). CARTIGRAFÍA DIGITAL Y CENSO DE EDIFICACIONES CABECERA MUNICIPAL DE ELREALEJO. MANAGUA: WEB: <HTTP://WWW.BCN.GOB. NI>.

Benita. (20 de Febrero de 2005). El Convento san FrancisCo. (R. G. VÁsquez, ENTREVISTAdor)

Bolaños, A. V. (1954). Documentos para la historia DE NicAragua, Colección Vega Boaños. Tomo VI. Pag. 121. Managua: Banco de America.

García, R. ( 2010). InVESTIGACIONES ARqueohistóricas en León Viejo, Primer Emplazamiento Colonial de NICARAGUA (1524-1610)PATRIMONIO DE LA HUMANIDAD. CONTRIBUCIONES REVISTA SAC CIENCIA-ARTE Y CULTURA, 6-18. turismo comunitario en El Realejo es necesario conocer lo sagrado, el mito, la ritualidad, el símbolo, el imaginario, la arquitectura, la gastronomía. La propuesta sería construir la "fantasía" del pueblo mágico, pero estudiando los haberes y saberes locales para que no se dañe la integridad comunitaria y para que sean ellos quienes reciban los beneficios. Debemos de tener muy en cuenta que descolonizando nuestros pensamientos, nuestras ideas, podemos llegar alcanzar una verdadera independencia cultural y económica con proyectos de emprendimientos ya sean macros o micros que coadyuven al buen vivir de las comunidades, y aporten al desarrollo socioeconómico de la nación.

HARRIS, M. (2006). ANTROPOLOGÍA CULTURAL. EEUU: S.E.

MelÉNdez, C. (1976). HeRnÁNdez de Córdoba, CaptTÁn de conquista de Nicaragua. Managua: Fondo de Promoción Cultural. Banco de America.

Montoya, R. (12 de Marzo de 2016). El Puerto de ICACo. (R. GARCÍA, ENTREVISTADOR)

Newson, L. A. (1986). Indian SuRVIVAL in Colonial NicaraGUA. OKLAHOMA: UNIVERSIDAD OKLAHOMA.

PÉREZ, C. A. (20 DE FEBRERo DE 2005). EL NOMBRE DE LA COMUNIDAD. (S. ESPINOZA, ENTREVISTADOR)

SÁNCHEZ, M. R. (1977). EL CoRREGIMIENTO de El REALEJO. EN M. R. SÁnchez, Historial de El Realejo (pág. 511). MANAGUA: SAN JOSÉ.

SÁnchez, M. R. (1977). Historial de El Realejo. Managua: SAN JOSÉ.

Zuniga, E. (1997). Historia EscleSIASTICA DE NicARAGUA. MANAGUA: UNIÓN. 


\section{Ramiro García Vásquez}

Realizó estudios de Licenciatura en Ciencias de la Educación con Mención en Biología (UNAN-Managua). Posteriormente hizo estudios Técnicos en el extranjero (México, Panamá, Suecia, Dinamarca) se especializó en Paleontología, Arqueología y Antropología Física. Entre 1994 y 2013 se desempeñó como Responsable del Departamento de Investigaciones Antropológicas del Museo Nacional de Nicaragua, realizó investigaciones paleontológicas, antropológicas y arqueológicas a nivel nacional. En el año 2001 fue condecorado con la Medalla Diocleciano Chávez y Las Llaves del Museo Nacional de Nicaragua en reconocimiento a su labor científica. Es miembro Honorario de la Academia de Geografía e Historia de Nicaragua. En los últimos 15 años se ha desempeñado como Consultor Cultural a nivel nacional e internacional.

\section{Sandra Verónica Espinoza}

Realizó estudios de Licenciatura en Administración turística (1999), posteriormente (2002) estudió un Técnico Superior en el Instituto Nacional Tecnológico de Nicaragua en Administración de la Cultura. Entre 2009 y 2010 hizo un Diplomado en Pedagogía de la Formación Profesional en la Universidad de Don Bosco, San Salvador, El Salvador. De 2000 a 2003 se desempeñó como Subdirectora del sitio León Viejo (Patrimonio Mundial). Del 2006 al 2014 fue directora del Museo Chorotega-Nicarao (Chinandega). De 2005 al 2014 trabajó como docente de Área Técnica o Formación Profesional. A partir del 2005 empezó a trabajar como consultora en temas turísticos, culturales y sobre liderazgo. Actualmente está cursando la Maestría en Antropología y Liderazgo Social. 